\title{
INJECTIVE COGENERATOR RINGS AND A THEOREM OF TACHIKAWA. II
}

\author{
CARL FAITH
}

ABSTRACT. The main theorem states that a right injective cogenerator ring $R$ has strongly bounded basic ring $R_{0}$, that is, every onesided ideal $\neq 0$ of $R_{0}$ contains an ideal $\neq 0$. A right injective cogenerator ring $R$ is characterized by the condition:

(1) $R$ is semiperfect and right self-injective and

(2) $R$ has (finite) essential right socle.

We show that (2) can be replaced by

( $\left.2^{\prime}\right) R_{0}$ is strongly right bounded and has finite left socle.

Actually a right perfect right self-injective ring $R$ is right PF iff $R_{0}$ is strongly bounded (Corollary 4).

We say that a module $M$ has $\mathrm{F}(\mathrm{ES})$ if $M$ has (finite) essential socle. Thus socle $M$ (= sum of the minimal submodules) is essential when $M$ has ES, and if $M$ has FES, then socle $M$ is a sum of finitely many simple modules. The Goldie dimension of a module $M$ is the maximum number (= integer) $n$ of nonzero independent submodules of $M$, when such exists. Then we write $\operatorname{dim} M=n$. (This is unambiguous by the Krull-Schmidt Theorem applied to the injective hull of $M$.) We introduce Goldie dimension in order to formalize several statements below. Note, however, that ES plus Goldie dimension $n<\infty \Rightarrow$ (F)ES

THEOREM 1. For a semiperfect right self-injective ring $R$ with basic ring $R_{0}$ the f.a.e.:

(1) (a) $R$ is right $P F(=R$ is an [injective] cogenerator in mod- $R)$.

(1) (b) $R$ has right $E S$.

(2) $R_{0}$ is strongly bounded with FES socle $S=V_{1} \oplus \cdots \oplus V_{n}$, where $V_{i}$ is an ideal which is a simple right, and a simple left, $R_{0}$-module, $i=1, \ldots, n<\infty$.

(3) $R_{0}$ is strongly bounded with left ES.

(4) $R_{0}$ is strongly right bounded and every simple left $R_{0}$-module $V$ embeds in $R_{0}$ so that $V R_{0}$ has finite length as a left $R_{0}$-module.

(5) $R_{0}$ is strongly right bounded, and every simple left $R_{0}$-module $V$ of $R_{0}$ embeds in $R_{0}$, and $V R_{0}$ contains a minimal ideal.

(6) $R_{0}$ is strongly right bounded with left ES, and every minimal left ideal $V$ generates an ideal $R_{0}$ of finite left Goldie dimension.

(7) $R_{0}$ is strongly right bounded, and every simple left $R$-module $V$ embeds in $R$

Received by the editors April 15, 1976.

AMS (MOS) subject classifications (1970). Primary 16A36, 16A52; Secondary 16A49, 16 A64.

- American Mathematical Society 1977 
so that $V R$ has finite length as a left $R$-module (or contains a minimal ideal of $R$ ).

(8) $R_{0}$ is strongly right bounded. $R$ has left $E S$, and every minimal left ideal $V$ of $R$ generates an ideal $V R$ of finite left length.

(9) $R_{0}$ is strongly bounded, and $R$ has left $E S$.

Proof. Now by the equivalent conditions $\left(\mathrm{PF}_{1}\right)-\left(\mathrm{PF}_{5}\right)$ stated in the Introduction of [1], we have $1(\mathrm{a}) \Leftrightarrow 1(\mathrm{~b})$.

(1) $\Rightarrow(2) . R_{0}$ is strongly right bounded by [1, Theorem 1$]$. In order to prove that $R$ has FES, it suffices to prove that $R_{0}$ has left FES inasmuch as $R_{0} \sim R$ (similarity or Morita equivalence as defined in [1]), and the condition (F)ES is characterizable in $R$-mod by the property that finitely generated projective modules have (F)ES. Hence assume $R=R_{0}$ (self-basic). Then $R / \operatorname{rad} R$ is a product of fields, hence every maximal right ideal is an ideal. Since $R$ is right self-injective, then every finitely generated left ideal $V$ of $R$ is a left annulet. Thus, $V$ satisfies $V={ }^{\perp}\left(V^{\perp}\right)$, and $V^{\perp}$ is contained in a maximal (right) ideal $P$. Since $R$ is right PF, every right ideal is a right annulet, so that ${ }^{\perp} P \neq 0$. Hence $V={ }^{\perp}\left(V^{\perp}\right)$ contains an ideal ${ }^{\perp} P \neq 0$. This proves that $R$ is strongly (left) bounded, and, moreover, that any simple left ideal $V$ is an ideal. Since $R$ is strongly right bounded by [1, Theorem 1$]$, then $V$ is a simple right ideal. Similarly, every simple right ideal is a simple left ideal. Thus the left socle $S_{l}=S_{r}$, the right socle. Since $R$ has right FES by $\mathrm{PF}_{2}$, then $S=S_{r}=S_{l}$ $=W_{1} \oplus \cdots \oplus W_{n}$ for finitely many ideals $W_{i}$ which are simple in $R$-mod and in mod- $R, i=1, \ldots, n$. Let $L \neq 0$ be a left ideal. Since $R$ is strongly left bounded then $L$ contains an ideal $\neq 0$, so that $L \cap S \neq 0$, proving that $R$ has left FES. This completes the proof of $(1) \Rightarrow(2)$.

$(2) \Rightarrow(3)$ is trivial.

(3) $\Rightarrow(4)$. Let $R=R_{0}$. If $V$ is any simple left ideal, then $V$ is an ideal, hence $V R=V$ has length $=1$. As in [1], we write $R=\oplus_{i=1}^{n} e_{i} R=R_{0}$ for right prindecs $e_{i} R, i=1, \ldots, n$. Let $B_{i}$ be an ideal $\neq 0$ contained in $e_{i} R$, and using the fact that $R$ has left ES, let $W_{i}$ be a simple left ideal contained in $B_{i}, i=1, \ldots, n$. Since $W_{i}$ is an ideal, and a minimal left ideal, and since $R$ is right bounded, then $W_{i}$ is a minimal right ideal. Moreover, since $R$ is right selfinjective, an isomorphism $W_{i} \approx W_{j} \Rightarrow \exists a \in R$ with $a W_{i}=W_{j}$ so $W_{i} \approx W_{j}$ for any $i \neq j$. Furthermore, $e_{i} R$ is an indecomposable injective, hence uniform, so that $W_{i}$ is essential in $e_{i} R$, and hence $W_{1} \oplus \cdots \oplus W_{n}$ is essential in $R$. This proves that the socle of $R_{R}$ has length $=n$ (hence by injectivity and selfbasicness of $R$ every simple right module $\hookrightarrow R$ ) so $R$ is right PF by $\mathrm{PF}_{3}$ (or $\mathrm{PF}_{5}$ ). Then, every simple left module $V \hookrightarrow R$ by [1, Corollary 11]. This completes the proof of $(3) \Rightarrow(4)$.

(4) $\Rightarrow(5)$ is trivial.

(5) $\Rightarrow$ (1). We may assume $R=R_{0}=\oplus_{i=1}^{n} e_{i} R$ as above. Also let $V_{1}, \ldots$, $V_{n}$ be the simple left modules of $R$ assumed to be embedded in $R$, and let $B_{i}$ be a minimal ideal $\neq 0$ contained in $V_{i} R, i=1, \ldots, n$. Since $R$ is strongly right bounded, $B_{i}$ is actually a minimal right ideal, and by right self-injectivity, 
we have $B_{i} \approx B_{j}$ for any $i \neq j, i=1, \ldots, n$. Thus, every simple right module $W \hookrightarrow R$, so that $R$ is $\left(\mathrm{PF}_{5}\right)$.

(6) $\Rightarrow(1)$. Since $R_{0}=R$ is strongly right bounded, then every $e_{i} R$ contains an ideal $B_{i} \neq 0$. Since $R$ has left ES, $B_{i}$ contains an ideal $W_{i}=V_{i} R$, where $V_{i}$ is a minimal left ideal $\neq 0, i=1, \ldots, n$. By the fact that $V_{i} R$ has finite left Goldie dimension, $W_{i}=V_{i} R$ is a finite direct sum of simple left ideals, and hence $V_{i} R$ has d.c.c. on left $R$-submodules, $i=1, \ldots, n$. Then, there is a minimal ideal $B_{i}$ of $R$ contained in $W_{i}$, and strong right boundedness of $R$ implies that $B_{i}$ is a minimal right ideal, $i=1, \ldots, n$. Moreover, $B_{i} \approx B_{j}, i$ $=1, \ldots, n$, so every simple right $R$-module embeds in $R=R_{0}$. Since $e_{i} R$ is injective, $B_{i}$ is essential in $e_{i} R, i=1, \ldots, n$, so $R$ has right (F)ES. This completes the proof of $(6) \Rightarrow(1)$.

(1) $\Rightarrow(6)$ via $(1) \Rightarrow(4) \Rightarrow(6)$.

(4) $\Leftrightarrow$ (7) (or (5) $\Leftrightarrow$ (7)) follows from the similarity $R_{0} \sim R$, which results from the category equivalence $T: R_{0}-\bmod \approx R-\bmod$ where $T \approx R e_{0} \otimes_{R_{0}}$ and $T^{-1} \approx e_{0} R \otimes_{R}$. To see this note that any left $R_{0}$-module $V_{0}$ is mapped onto $V=R e_{0} \otimes_{R_{0}} V_{0}$, and that $V$ is simple iff $V_{0}$ is simple. Moreover, every simple left $R$-module $V$ comes from a simple $V_{0}=T^{-1} V$. Furthermore, $V_{0} \hookrightarrow R$ implies by exactness of $T$ (= flatness of $R e_{0}$ in mod- $R_{0}$ ) that $V \approx R e_{0} V_{0}$ $=R V_{0} \hookrightarrow R$. Thus, $V \hookrightarrow R$ for all simple $V \in R$-mod iff $V_{0} \hookrightarrow R$ for all simple $V_{0} \in R_{0}$-mod .

To continue with (4) $\Rightarrow(7)$, we have that (4) $\Rightarrow(2)$ which by the remark made in the proof of $(1) \Rightarrow(2)$ implies every finitely generated projective left $R$-module has FES. Thus, $R V=$ a direct sum of simple left ideals $\approx V$.has finite length.

To complete the equivalence of $(4) \Leftrightarrow(7)$, we note that (5) $\Leftrightarrow$ parenthetic part of (7) inasmuch as the Morita theorems imply that the correspondence

$$
\text { ideals } \begin{aligned}
R_{0} & \rightarrow \text { ideals } R, \\
I_{0} & \mapsto I=R I_{0} R, \\
e_{0} I e_{0} & \leftrightarrow I,
\end{aligned}
$$

is a bijection. Thus, since $V_{0} R_{0}$ maps into $V R$ under this correspondence, we have the stated equivalence.

Now (7) $\Rightarrow(4)$ can be proved since (7) $\Rightarrow V R$ contains a minimal ideal $B$ which implies that $V_{0} R_{0}$ contains a minimal ideal $B_{0}$, so (7) $\Rightarrow(5)$, and (5) $\Rightarrow$ (4).

(6) $\Leftrightarrow(8)$ inasmuch as $R_{0}$ has left ES iff $R$ has left ES. (This remark also suffices for (3) $\Leftrightarrow(9)$.) Then the equivalence of (6) and (8) follows as the equivalence of (4) and (7). (Note $V R$ has finite length iff it has finite Goldie dimension.)

2. CoRollary. A semiperfect right FPF ring $R$ with nil radical is right $P F$ iff any of the equivalent conditions hold:

2(a). $R$ has finite essential left socle. 
2(b). Every simple left $R$-module $V$ embeds in $R$, and $V R$ has finite length as a left $R$-module (or else VR contains a minimal ideal).

Proof. $R$ is right self-injective and $R_{0}$ is strongly right bounded by [ 1 , Theorem 1]. Then (2) and (3) of Theorem 1 apply for (a), and (b) is equivalent to (7).

3. CoROllary. A right perfect right FPF ring $R$ is right PF iff $R$ satisfies any of the following equivalent conditions:

(a) $R$ has finite left Goldie dimension.

(b) Every simple left ideal generates an ideal of finite left length.

(c) $R$ has finite left socle.

(d) Every ideal of $R$ contains a minimal ideal.

Proof. A right perfect ring $R$ has left ES and nil radical so 2(a) $\Leftrightarrow 3$ (a) and (c) $\Leftrightarrow$ (a). Also 2(b) $\Leftrightarrow 3(\mathrm{~b})$. Clearly $3(\mathrm{~d}) \Rightarrow 2(\mathrm{~b})$ (the parenthetical part), and conversely if every $V R$ contains a minimal ideal, then so does every ideal $I$. $\neq 0$, since $I$ contains a minimal left ideal $V$.

4. Corollary. A right self-injective right perfect ring $R$ is right $P F$ iff the basic ring $R_{0}$ is (right and left) strongly bounded.

Proof. $R$ right perfect $\Rightarrow R_{0}$ right perfect $\Rightarrow R_{0}$ has left ES so the corollary is statement (3) of the theorem.

For emphasis, we deduce a theorem of Osofsky and Utumi (for $R_{R}$ injective) and Tachikawa (for $R_{R}$ FPF):

5. COROLLARY. Any right self-injective or right FPF left perfect ring $R$ is right $P F$ (and $R_{0}$ is strongly bounded).

Proof. Right FPF left perfect is right self-injective. In this case, $R$ has right ES, so $R$ is right PF (by $1(\mathrm{~b})$ ), and $R_{0}$ is strongly bounded by Theorem $1(2)$.

Acknowledgement. The author wishes to thank Professor Kent R. Fuller for raising the question about a "theorem" originally stated in [1] which led to the fuller investigation in this paper. The "theorem" is still a reasonable conjecture in view of Corollary 4; namely, is a right perfect right FPF ring right PF?

\section{REFERENCES}

1. C. Faith, Injective cogenerator rings and a theorem of Tachikawa, Proc. Amer. Math. Soc. 60 (1976), 25-30.

Department of Mathematics, Rutgers University, New Brunswick, New Jersey 08903 